\title{
Establishment and characterization of monoclonal 5-fluorouracil-resistant cell lines derived from human endometrial adenocarcinoma
}

\author{
TETSUJI TANAKA, TAO BAI and SAORI TOUJIMA
}

Department of Obstetrics and Gynecology, Wakayama Medical University, 811-1 Kimi-idera, Wakayama 641-0012, Japan

Received March 10, 2010; Accepted May 6, 2010

DOI: 10.3892/ijo_00000722

\begin{abstract}
To investigate acquired 5-fluorouracil (5FU)resistance in cancer cells, we established four monoclonal 5FU-resistant cell lines from human endometrial adenocarcinoma cells by long-term 5FU-exposure cultures and limiting dilution cultures. The established subclones exhibited 5-25 times greater 5FU-resistance than the parent cells, and showed suppression of 5FU-induced DNA fragmentation. All four 5FU-resistant subclones were 25-125 times more resistant to SN38, 4-hydroxy-cyclophosphamide, paclitaxel and etoposide than the parent cells while none of the four subclones showed resistance to mitomycin. Two of the four subclones showed no resistance to pirarubicin and bleomycin. Although all four 5FU-resistant subclones were 5-25 times more resistant to anti-Fas IgM than the parent cells, the resistance levels to anti-Fas IgM of the individual subclones did not coincide with the strengths of their multidrug resistance. Karyotyping analyses revealed that the parent cells and the three 5FUresistant subclones examined had normal 46XX karyotypes. These results indicate that the cell death signals induced by mitomycin, pirarubicin and bleomycin are distinctly different from the Fas-mediated apoptotic signals, that acquisition of 5FU-resistance can occur without any large chromosomal deletions or rearrangements, and that there are several possible molecular changes during the acquisition of 5FUresistance. The established cell lines represent useful tools for investigating the mechanisms and treatments of acquired 5FU-resistance.
\end{abstract}

\section{Introduction}

Recent anticancer chemotherapies have improved the overall survival rates of advanced cancer patients. 5-Fluorouracil

Correspondence to: Dr Tetsuji Tanaka, Department of Obstetrics and Gynecology, Wakayama Medical University, 811-1 Kimi-idera, Wakayama 641-0012, Japan

E-mail: obgywmu@wakayama-med.ac.jp

Key words: fluorouracil, drug-resistance, Fas, apoptosis, karyotype, endometrial adenocarcinoma
(5FU) is often administered to advanced colorectal cancer patients, but their long-term survival rates have not yet improved. However, a recent combination chemotherapy with 5FU and oxaliplatin has brought about a remarkable improvement of the long-term survival rates of inoperable colorectal cancer patients (1). Consequently, this combination chemotherapy with $5 \mathrm{FU}$ and oxaliplatin has also been applied to recurrent ovarian cancer patients after standard chemotherapy with paclitaxel and carboplatin $(2,3)$. Since $5 \mathrm{FU}$-combination chemotherapies have been reapplied to various cancer patients in addition to colorectal cancer patients, 5FU-resistance may become a large clinical problem in future cancer chemotherapies. Although many reports have established that drugresistant cancer cells show cross-resistance to 5FU, few reports have indicated that novel 5FU-resistant cell lines were originally established. Moreover, there are few recent basic studies regarding the mechanisms of acquired 5FUresistance and cross-resistance to 5FU and other new anticancer drugs. Investigations of the mechanisms of 5FUresistance can lead to the development of novel effective anticancer chemotherapies for 5FU-resistant patients.

The present first-line chemotherapy for ovarian cancer and endometrial cancer is a combination chemotherapy with a taxane compound and a platinum compound, usually paclitaxel and carboplatin (TC therapy). Combination chemotherapy with $5 \mathrm{FU}$ and oxaliplatin was reported to be effective for recurrent ovarian cancer patients after TC therapy $(2,3)$. Therefore, 5FU-resistance may become a large clinical problem for endometrial and ovarian cancer patients in the near future. To investigate the mechanisms involved in the acquisition of 5FU-resistance, we established monoclonal 5FU-resistant cell lines from the human endometrial adenocarcinoma cell line HHUA (4) in this study.

\section{Materials and methods}

Anticancer drugs. All anticancer drugs were kind gifts from pharmaceutical companies. Mitomycin C (MMC) and 5FU were provided by Kirin-Kyowa-Hakko Co., Ltd. (Tokyo, Japan). SN38, a major active metabolite of irinotecan-HCl (CPT-11), was obtained from Yakult Honsha Co., Ltd. (Tokyo, Japan). Paclitaxel (PTX) and etoposide (VP16) were provided by Bristol-Myers Squibb Japan Co., Ltd. (Tokyo, Japan). Pirarubicin-HCl (THP) was provided by Meiji-Seika Kaisha Ltd. (Osaka, Japan). Cisplatin (CDDP) and bleomycin (BLM) 
were provided by Nihon-Kayaku Co., Ltd. (Tokyo, Japan). Nedaplatin (NPL) and 4-hydroxy-cyclophosphamide (4OHCPM), a major active metabolite of cyclophosphamide, were obtained from Shionogi \& Co., Ltd. (Osaka, Japan).

Cell line and culture. The HHUA cell line (4) was obtained from the Riken Cell Bank (Tsukuba, Japan). The cells were cultured in OPTI-MEM (Invitrogen Corp., Carlsbad, CA) supplemented with $5 \%$ fetal bovine serum (FBS) (Equitech Bio Inc., Ingram, TX), penicillin (PC) (100 U/ml) (Invitrogen) and streptomycin $(\mathrm{SM})(100 \mathrm{U} / \mathrm{ml})$ (Invitrogen Corp.) under $5 \% \mathrm{CO}_{2}$ and $95 \%$ air at $37^{\circ} \mathrm{C}$.

Establishment of 5FU-resistant subclones from HHUA cells. To establish 5FU-resistant subclones, HHUA parent cells were cultured with various concentrations of 5FU for 3-5 weeks, and the surviving cells were collected. This collection procedure after 5FU exposure was repeated four times. Finally, four single cell-derived etoposide-resistant subclones, designated 5FUr-3C, 5FUr-3D, 5FUr-4A and 5FUr-10B, were established by the limiting dilution method (5). The monoclonality of the individual 5FU-resistant subclones was confirmed by chromosome analyses as described below. The establishment of these 5FU-resistant subclones took 12 months.

Anticancer drug-sensitivity and anti-Fas IgM-sensitivity assays. The effects of anticancer drugs and anti-Fas IgM on cell growth were assayed as follows. Cells in the log phase were detached with $0.25 \%$ trypsin/ $1 \mathrm{mM}$ EDTA (Invitrogen) and cultured overnight in 96 -well plates $\left(5 \times 10^{3}\right.$ cells/well). On day 2, various concentrations of anticancer drugs or anti-Fas IgM (clone CH-11; MBL, Nagoya, Japan) were added to the cells. On day 4 , the numbers of viable cells were evaluated using a cell proliferation assay kit (Dojin Corp., Tokyo, Japan) and expressed as the percentages of viable cells relative to the mean number of viable untreated cells. All experiments were performed in triplicate. Data are shown as the mean $\pm \mathrm{SD}$, and comparative data were analyzed by Student's t-test $(n=6)$ and ANOVA.

DNA fragmentation assay. HHUA parent cells and 5FUresistant subclones in the log phase were detached with $0.25 \%$ trypsin/1 mM EDTA and cultured overnight in culture dishes ( $3 \times 10^{6}$ cells/dish) containing OPTI-MEM/5\% FCS/PC/SM. On day 2, 5FU (final concentrations: 3.37 and $16.7 \mu \mathrm{g} / \mathrm{ml}$ ) was added to the cells. On day 4, genomic DNA was extracted from all cells, including the dead ones, using a SepaGene DNA extraction kit (Sankyo-Junyaku Co., Ltd., Tokyo, Japan) and treated with $100 \mu \mathrm{g} / \mathrm{ml}$ of RNase A (Sigma Chemical Co., St. Louis, MO) in TE buffer (10 mM Tris, $\mathrm{pH} 8.0,2 \mathrm{mM}$ EDTA) for $90 \mathrm{~min}$ at $37^{\circ} \mathrm{C}$ to remove any contaminating RNA. Next, $\sim 20 \mu \mathrm{g}$ of the genomic DNA isolated from $5 \times 10^{5}$ cells was electrophoresed in a $1.4 \%$ agarose gel at $50 \mathrm{~V}$ for $\sim 2 \mathrm{~h}$, stained with $5 \mu \mathrm{g} / \mathrm{ml}$ of ethidium bromide and visualized by UV fluorescence.

Karyotyping analysis. Cytogenetic analyses were performed according to previously reported methods $(6,7)$ with the following modifications. Briefly, tumor cell cultures were washed and incubated with $0.1 \%(\mathrm{v} / \mathrm{v})$ colcemide (Sigma

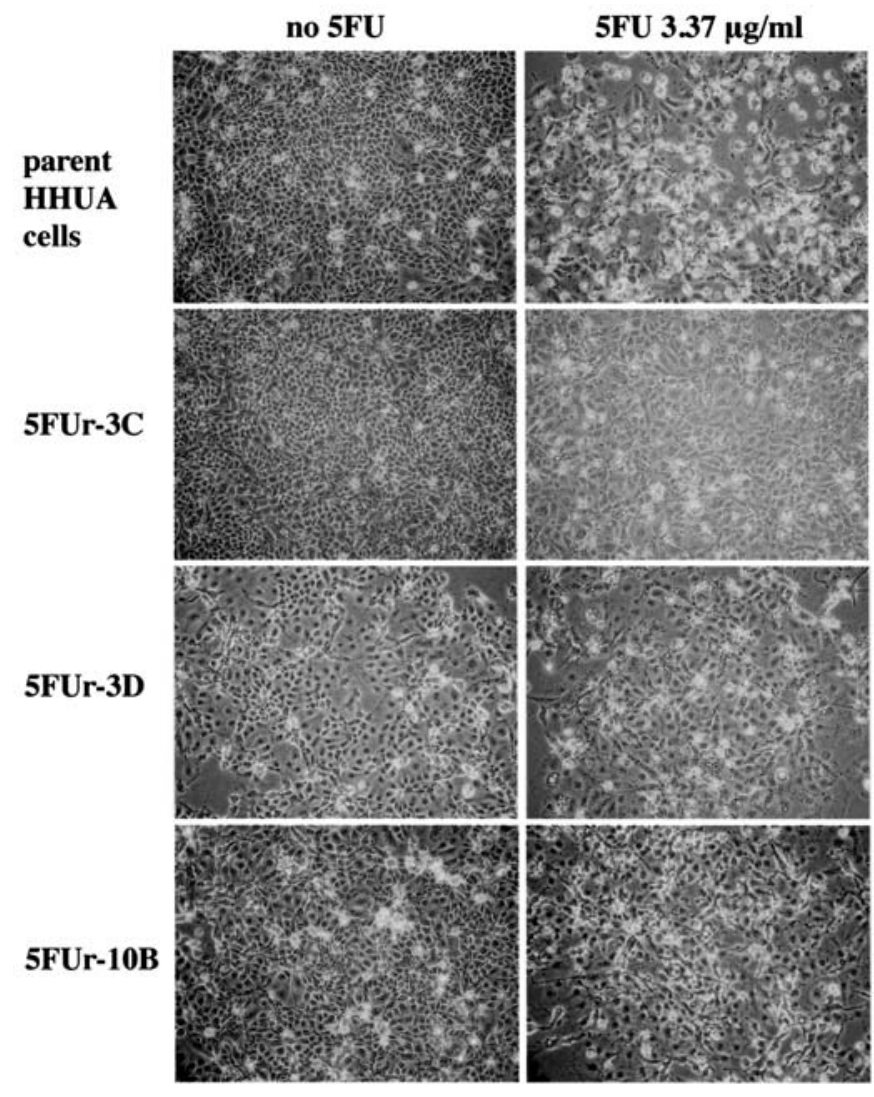

Figure 1. Microscopic images of cultured parent cells and three 5FUresistant subclones. The figures on the the left show cells cultured without $5 \mathrm{FU}$ for 2 days while the figures on the right show cells cultured with 5FU at $3.37 \mu \mathrm{g} / \mathrm{ml}$ for 2 days.

Chemical Co.) overnight. The cells were then exposed to a hypotonic solution composed of $3 \mathrm{~g} / 1 \mathrm{KCl}, 0.2 \mathrm{~g} / 1$ EGTA and $4.8 \mathrm{~g} / \mathrm{l}$ HEPES. The cells were centrifuged into a pellet and fixed in a methanol solution. G-banding was performed using the method of Yunis (8).

\section{Results}

Establishment of 5FU-resistant cell lines. Four monoclonal 5FU-resistant subclones, 5FUr-3C, 5FUr-3D, 5FUr-4A and 5FUr-10B, were established independently by long-term 5FU-exposure cultures and limiting dilution cultures as described in Materials and methods. Microscopic views of their cellular appearance and their adherence to culture dishes could not discriminate them from those of the parent HHUA cells (Fig. 1). After treatment with 5FU, however, detached dead cells only increased in the parent HHUA cell cultures (Fig. 1). As shown in Fig. 2A, all four 5FU-resistant subclones exhibited $\sim 5-25$ times higher IC50s (50\% viabilityinhibitory concentrations) for 5FU-sensitivity than the parent cells. Moreover, the 5FU-resistant subclones showed resistance against 5FU-induced apoptotic cell death, as evaluated by DNA fragmentation assays (Fig. 2B).

Cross-resistance to other anticancer drugs. Anticancer drugsensitivity tests were performed on the four 5FU-resistant subclones to investigate the mechanisms involved in the acquired 5FU-resistance. Two of the four resistant subclones, 


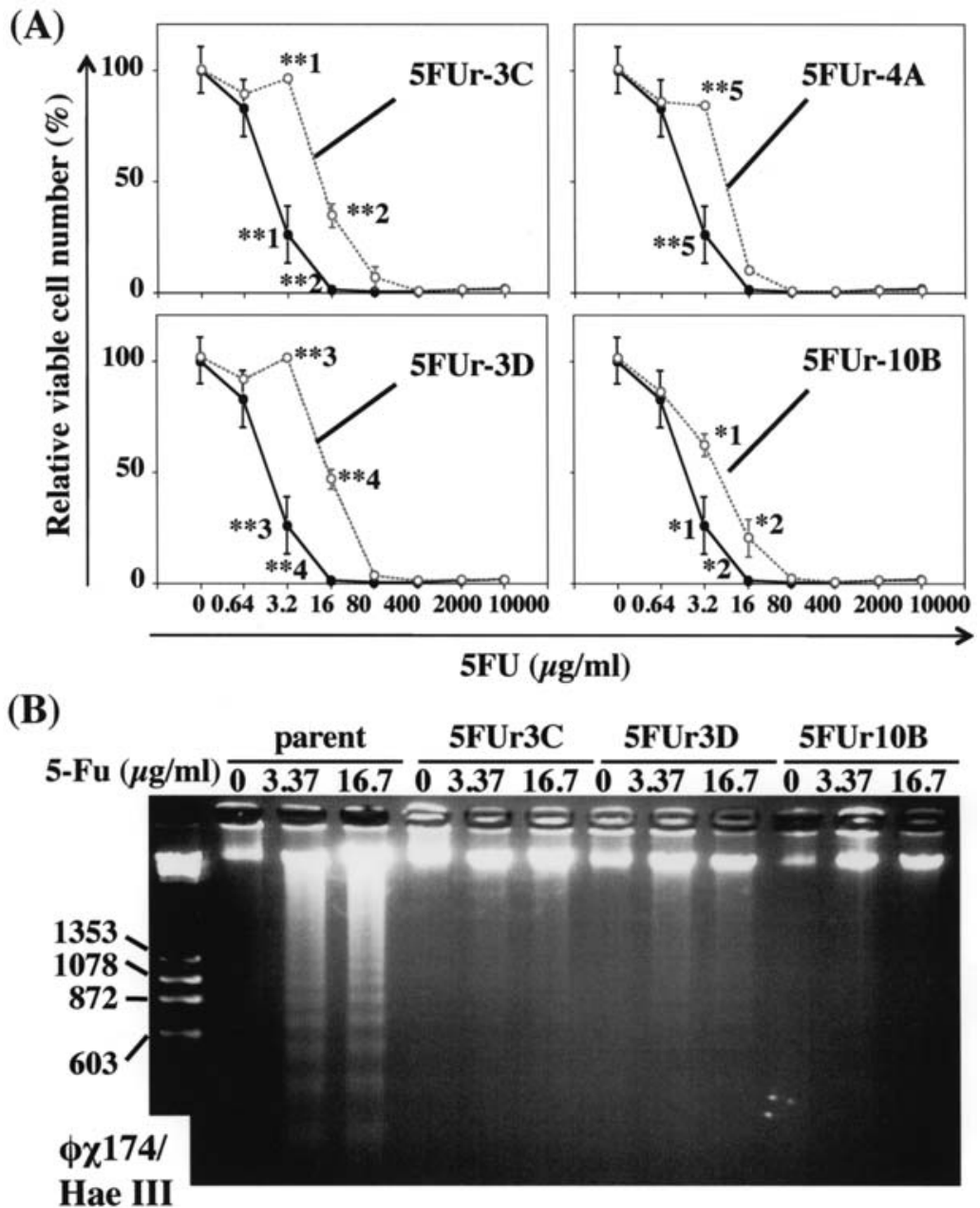

Figure 2. 5FU-sensitivity assays and DNA fragmentation assays of the 5FU-resistant subclones. (A) 5FU-sensitivity assays of the four 5FU-resistant subclones. The solid line with filled circles in each figure indicates the 5FU-sensitivity curve of the parent HHUA cells. The dotted lines with open circles show the 5FU-sensitivity curves of the 5FU-resistant subclones. ${ }^{*} 1$ and ${ }^{*} 2, \mathrm{p}<0.05$. ${ }^{* *} 1-{ }^{* *} 5, \mathrm{p}<0.01$. (B) DNA fragmentation assays of the $5 \mathrm{FU}-\mathrm{resistant}$ subclones.

5FUr-3C and 5FUr-3D, showed much more resistance to nine anticancer drugs other than 5FU compared with the remaining two subclones. Among the four subclones, 5FUr10B showed the least resistance against the 10 anticancer drugs. As shown in Fig. 3, all four 5FU-resistant subclones showed strong cross-resistance to SN38, 4OH-CPM, PTX and VP16 but none of the subclones showed cross-resistance to MMC. All four 5FU-resistant subclones were $>125$ times more resistant to $\mathrm{SN} 38$ than the parent cells. Three subclones, 5FUr-3C, 5FUr-3D and 5FUr-4A, exhibited $>125$ times higher IC50s for 4OH-CPM-sensitivity and PTX-sensitivity than the parent cells while subclone 5FUr-10B showed an $\sim 25$ times higher IC50. All four 5FU-resistant subclones showed 5-25 times higher IC50s for VP16-sensitivity than the parent cells. Mild increases in the IC50s for BLM-sensitivity and THP-sensitivity were observed in 5FUr-3C and 5FUr-3D while the other two subclones did not show apparent resistance to BLM and THP.

Anti-Fas IgM-sensitivity assays. The parent HHUA cells are well known to respond to specific Fas-mediated apoptotic signals stimulated by anti-Fas IgM treatment (9). To examine whether the four 5FU-resistant subclones acquired any resistance to Fas-mediated apoptotic signals, anti-Fas IgMsensitivity assays were performed. As shown in Fig. 4, subclones 5FUr-3C and 5FUr-10B exhibited $>25$ times higher IC50s for anti-Fas IgM-sensitivity than the parent cells while subclones 5FUr-3D and 5FUr-4A showed 5-25 times higher IC50s.

Karyotyping analyses. We carried out karyotyping analyses to examine the possibility that some major chromosomal changes during the establishment of the 5FU-resistant subclones were involved in the simultaneous acquisition of 5FUresistance, multidrug-resistance and anti-Fas IgM-resistance. The karyotyping analyses showed normal 46XX karyotypes in the 20 HHUA parent cells examined (Fig. 5). Moreover, the three 5FU-resistant subclones examined also revealed normal 46XX karyotypes (Fig. 5).

\section{Discussion}

$5 \mathrm{FU}$ is a major anticancer drug that is clinically used for patients with various types of cancers. In this study, we established and characterized four monoclonal 5FU-resistant subclones from the drug-sensitive endometrial adenocarci- 


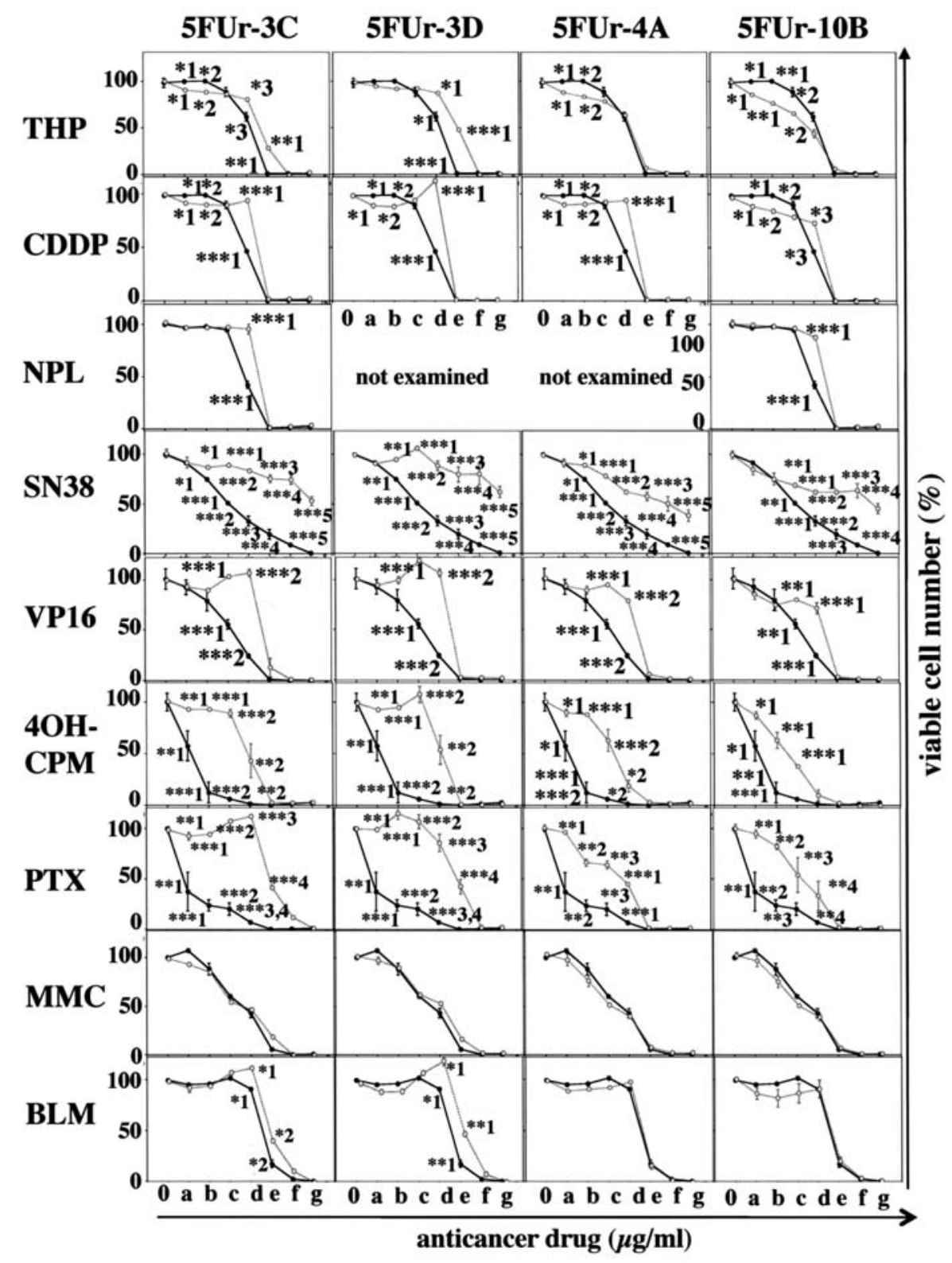

Figure 3. Anticancer drug-sensitivity assays of the four $5 \mathrm{FU}$-resistant subclones. The final concentrations $(\mu \mathrm{g} / \mathrm{ml})$ of each anticancer drug indicated as 0 and (a-g) at the bottom of the figures were as follows: THP: $0,0.001067,0.00533,0.0267,0.133,0.67,3.33$ and $16.670 \mu \mathrm{g} / \mathrm{ml}$; CDDP: $0,0.032,0.16,0.8,4,20$, 100 and $500 \mu \mathrm{g} / \mathrm{ml}$; NPL: 0, 0.0256, 0.128, 0.64, 3.2, 16, 80 and $400 \mu \mathrm{g} / \mathrm{ml} ; \mathrm{SN} 38: 0,0.00256,0.0128,0.064,0.32,1.6,8 \mathrm{and} 40 \mu \mathrm{g} / \mathrm{ml} ; \mathrm{VP} 16: 0,0.256,1.28$, $6.4,32,160,800$ and $4000 \mu \mathrm{g} / \mathrm{ml}$; 4OH-CPM: 0, 0.32, 1.6, 8, 40, 200, 1000 and $5000 \mu \mathrm{g} / \mathrm{ml}$; PTX: 0, 0.0128, 0.064, 0.32, 1.6, 8, 40 and $200 \mu \mathrm{g} / \mathrm{ml}$; MMC: 0 , $0.128,0.064,0.32,1.6,3.2,40$ and $200 \mu \mathrm{g} / \mathrm{ml}$; BLM: 0, 0.32, 1.6, 8, 40, 200, 1000 and $5000 \mu \mathrm{g} / \mathrm{ml} .{ }^{*} 1{ }^{*} 3, \mathrm{p}<0.05 .{ }^{* * *} 1{ }^{* *} 4, \mathrm{p}<0.01{ }^{* * *} 1-^{* * * *} 5, \mathrm{p}<0.001$.

noma cell line HHUA that expresses functional Fas antigen (9). To the best of our knowledge, this is the first report to investigate the interactions of 5FU-resistance and Fasmediated apoptosis in human endometrial adenocarcinoma cells. The established 5FU-resistant subclones showed reduced susceptibility to Fas-mediated apoptosis and crossresistance to eight other anticancer drugs in addition to 5FU. However, the levels of reduction of the Fas-mediated apoptosis did not coincide with the multidrug-resistance among the four 5FU-resistant subclones. These findings suggest that the 5FU-resistant subclones may have some impaired cell signaling processes that are common to cell death signals induced by various anticancer drugs and Fasstimulation. The established 5FU-resistant subclones will be useful for identifying efficient chemotherapy protocols for advanced cancer patients with resistance to standard chemotherapies with $5 \mathrm{FU}$. We are now investigating the common signals, which are considered to be impaired in the established 5FU-resistant subclones, between anticancer drug-induced apoptotic signals and Fas-mediated cell death signals.

It is noteworthy that none of the established 5FU-resistant subclones showed cross-resistance to MMC. Moreover, the 5FUr-4A and 5FUr-10B subclones did not acquire crossresistance to THP and BLM while the other two 5FU-resistant subclones, 5FUr-3C and 5FUr-3D, exhibited mild crossresistance to THP and BLM. These results indicate that the cell death signals activated by MMC, THP and BLM do not have any common death signals with Fas-mediated apoptotic 


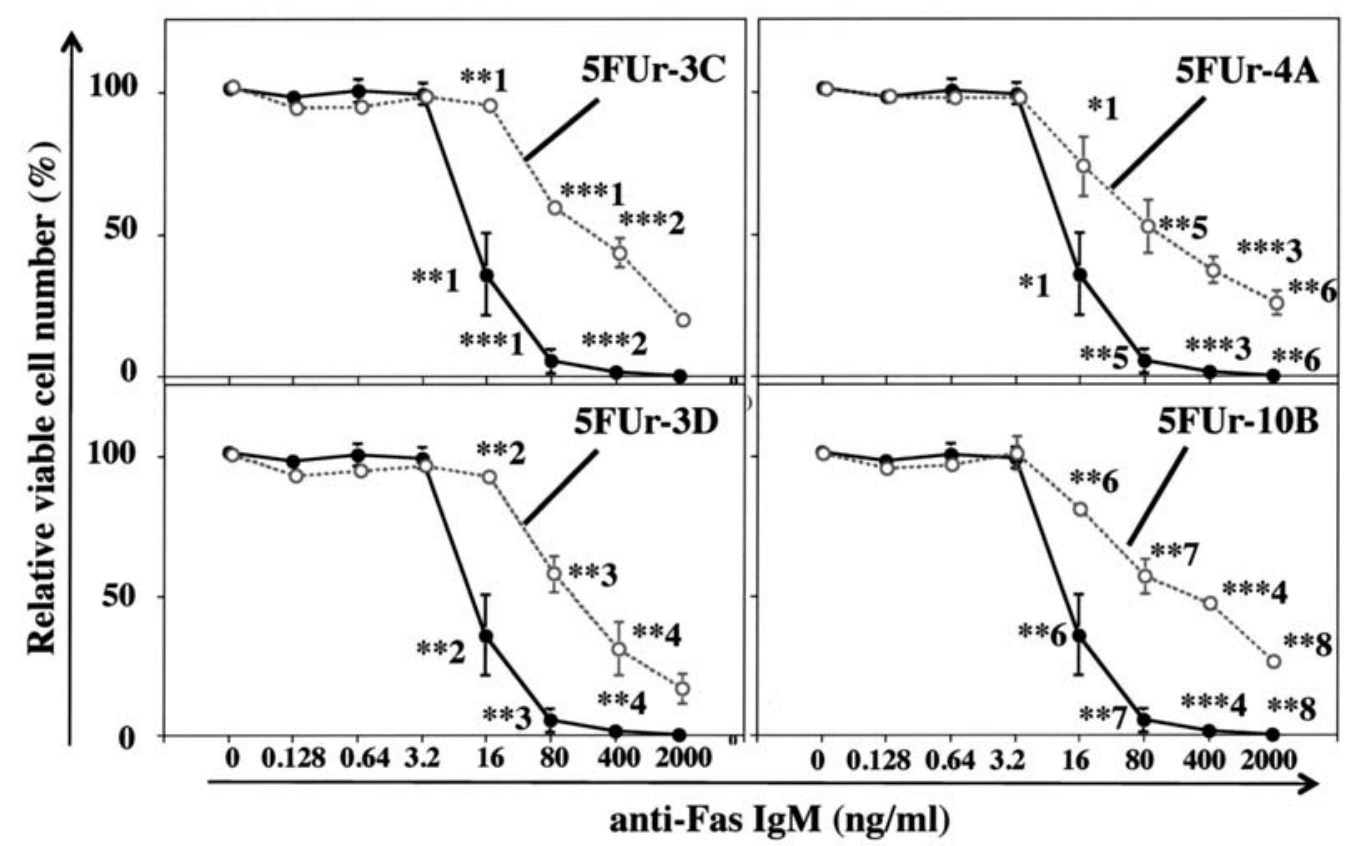

Figure 4. Anti-Fas IgM-sensitivity assays of the 5FU-resistant subclones. The solid line with closed circles in each figure indicates the anti-Fas IgMsensitivity curve of the parent HHUA cells. The dotted lines with open circles show the anti-Fas IgM-sensitivity curves of the 5FU-resistant subclones. ${ }^{*} 1$, $\mathrm{p}<0.05 .{ }^{* *} 1-^{* *} 8, \mathrm{p}<0.01 .{ }^{* * *} 1-^{* * *} 4, \mathrm{p}<0.001$.

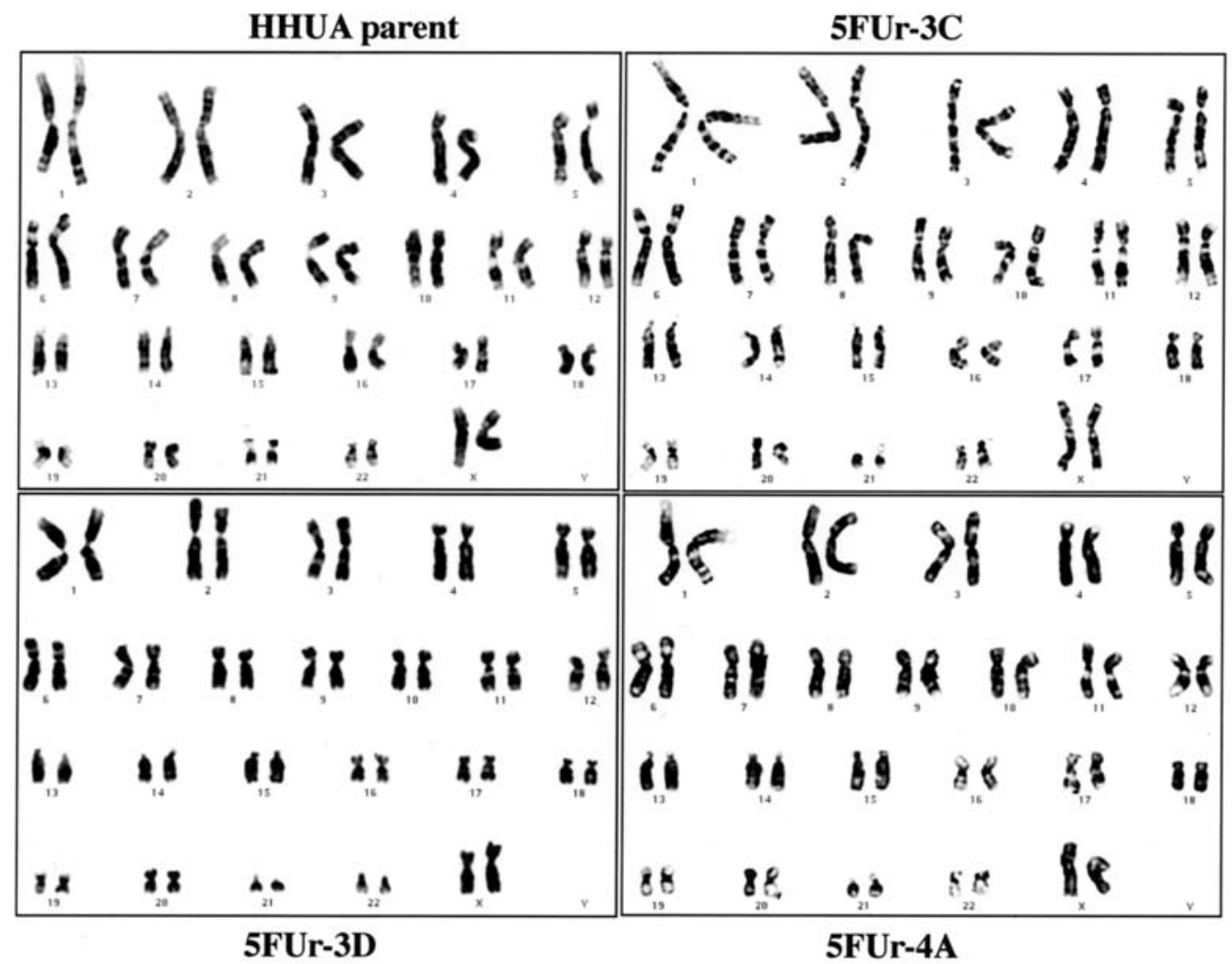

Figure 5. Karyotyping analyses of HHUA-derived 5FU-resistant subclones. The HHUA parent cells and the three 5FU-resistant subclones examined show normal 46XX karyotypes.

signals. In addition, the results suggest that chemotherapeutic regimens with MMC, THP or BLM may have possible antitumor effects in advanced cancer patients who have become resistant to 5FU-combined chemotherapies. Although all the established 5FU-resistant subclones showed mild resistance to CDDP, modified CDDP-combined chemotherapies may 
have possible clinical therapeutic effects in advanced 5FUcombined chemotherapy-resistant cancer patients. All four 5FU-resistant subclones showed strong resistance to SN38, VP16, 4OH-CPM and PTX, indicating that acquisition of 5FU-resistance may have some relationships with acquisition of resistance against DNA topoisomerases, which are the major targets of SN38 and VP16. The results also suggest that chemotherapies with CPT-11, VP16, CPM or PTX may not have clinically effective anticancer effects in advanced 5FU-combined chemotherapy-resistant cancer patients.

The parent HHUA cells are highly differentiated endometrial adenocarcinoma cells that express estrogen receptors and progesterone receptors similar to normal human endometrial epithelial cells (4). The most interesting findings in the present study may be that there were no major abnormalities in the karyotyping analyses of the established subclones. Since the 5FU-resistant cells were multidrugresistant and Fas-insensitive, the results indicate that acquisition of 5FU-resistance with multidrug-resistance can be completed without any large chromosomal deletions or rearrangements. Therefore, investigation of the impaired cell death signals in the 5FU-resistant subclones established in this study may lead to a breakthrough in elucidating the multidrug-resistance of cancer cells.

\section{References}

1. De Gramont A, Figer A, Seymour M, Homerin M, Hmissi A, Cassidy J, Boni C, Cortes-Funes H, Cervantes A, Freyer G, Papamichael D, Le Bail N, Louvet C, Hendler D, De Braud F, Wilson C, Morvan F and Bonetti A: Leucovorin and fluorouracil with or without oxaliplatin as first-line treatment in advanced colorectal cancer. J Clin Oncol 18: 2938-2947, 2000.

2. Pectasides D, Pectasides M, Farmakis D, Gaglia A, Koumarianou A, Nikolaou M, Koumpou M, Kountourakis P, Papaxoinis G, Mitrou P, Economopoulos T and Raptis SA: Oxaliplatin plus high-dose leucovorin and 5-fluorouracil (FOLFOX 4) in platinum-resistant and taxane-pretreated ovarian cancer: a phase II study. Gynecol Oncol 95: 165-172, 2004.

3. Rosa DD, Awada A, Mano MS, Selleslags J, Lebrun F, Gil T, Piccart MJ and D'Hondt V: Oxaliplatin/5fluorouracil-based chemotherapy was active and well tolerated in heavily pretreated patients with ovarian carcinoma. Arch Gynecol Obstet 278: 457-462, 2008.

4. Ishiwata I, Ishiwata $\mathrm{C}$, Soma $\mathrm{M}$, Arai $\mathrm{J}$ and Ishikawa $\mathrm{H}$ : Establishment of human endometrial adenocarcinoma cell line containing estradiol-17 beta and progesteron receptors. Gynecol Oncol 17: 281-290, 1984.

5. Tanaka T, Nakajima S and Umesaki N: Cellular heterogeneity in long-term surviving cells isolated from eutopic endometrial, ovarian endometrioma and adenomyosis tissues. Oncol Rep 10: 1150-1160, 2003

6. Gibas Z, Prout GR, Conolly JG, Pontes JE and Sandberg AA: Non-random chromosome changes in transitional cell carcinoma of the bladder. Cancer Res 44: 1257-1264, 1984

7. Yoshida MA, Ohyashiki K, Ochi H, Gibas Z, Pontes JE, Prout GR Jr, Huben R and Sandberg AA: Cytogenetic studies of tumor tissue from patients with non-familial renal cell carcinoma. Cancer Res 46: 2139-2147, 1986.

8. Yunis JJ: New chromosome techniques in the study of human neoplasia. Hum Pathol 12: 540-549, 1981.

9. Tanaka $\mathrm{T}$ and Umesaki N: Cytokine regulation of apoptotic susceptibility in a human endometrial epithelial cell line. J Reprod Immunol 47: 105-119, 2000. 